\title{
VERSÃO PORTUGUESA DA ESCALA DE AUTO-AVALIAÇÃo RETROSPECTIVA DA INIBIÇÃO COMPORTAMENTAL: ESTUDO PSICOMÉTRICO DA RSRI NUMA AMOSTRA DE ADOLESCENTES DA COMUNIDADE
}

\author{
Marina Cunha ${ }^{1}$ \\ José Pinto-Gouveia ${ }^{2}$ \\ Catarina Morgado
}

Resumo: O conceito de inibição comportamental é definido como uma apreensão ou reserva consistente na forma de responder a estímulos sociais ou não-sociais novos e não familiares. Dada a inequívoca importância da avaliação da inibição comportamental na infância e de uma melhor compreensão do seu papel no desenvolvimento da psicopatologia, Reznick e colaboradores desenvolveram a escala Retrospective Self-Report Inhibition (RSRI), formada por 30 itens que procuram avaliar, retrospectivamente, o grau de inibição comportamental do individuo durante a infância (entre o $1^{\circ}$ e $6^{\circ}$ ano de escolaridade). O objectivo deste artigo é confirmar as qualidades psicométricas da RSRI aplicada a uma amostra da população portuguesa e investigar a utilidade deste instrumento enquanto medida da inibição comportamental expressa na infância. O primeiro estudo, realizado com uma amostra de adolescentes $(n=296)$, entre os 12 e os 18 anos, revelou que a RSRI possui uma boa consistência interna e uma estrutura factorial idêntica à versão original, mantendo-se a evidência de dois factores: medos/doenças, respeitante a situações relacionadas com medos, em geral, e queixas somáticas; e escola/situações sociais, que integra os itens relacionados com medos em contexto escolar e em diversas situações sociais. No segundo estudo, recorrendo a grupos clínicos e de controlo (Grupo de jovens com Perturbação de Ansiedade Social $(n=76)$, Outras Perturbações Ansiosas $(n=28)$ e Sem qualquer Psicopatologia $(\mathrm{n}=76)$ procurou-se analisar a capacidade discriminativa da escala e suas dimensões. Os resultados evidenciaram a utilidade bidimensional do constructo de inibição comportamental, tal como é avaliado pela RSRI, mostrando a dimensão de medos sociais mais poder discriminativo do que a dimensão de medos não-sociais.

\footnotetext{
${ }^{1}$ Instituto Superior Miguel Torga; Centro de Investigação do Núcleo de Estudos e Intervenção Cognitivo-Comportamental (CINEICC) da Universidade de Coimbra. Correspondência: marina_cunha@ismt.pt

${ }^{2}$ Universidade de Coimbra, Faculdade de Psicologia e de Ciências da Educação, Centro de Investigação do Núcleo de Estudos e Intervenção Cognitivo-Comportamental (CINEICC)

${ }^{3}$ Escola Superior de Educação de Coimbra
} 
São apontadas sugestões para futuros estudos que reforcem a robustez dos dados encontrados e contribuam para a utilidade desta escala na investigação clínica em adolescentes portugueses.

Palavras-chave: Escala RSRI, adolescência, questionários de auto-resposta, inibição comportamental, ansiedade social

The Portuguese version of the Retrospective Self-Report of Inhibition: a psychometric analysis of RSRI in an Adolescent Community Sample (Abstract): Behavioural inhibition is defined as a consistent restraint in response to unfamiliar social or nonsocial stimuli. Due to the unequivocal importance of behavioural inhibition assessment during childhood and to a better understanding of its role in the development of psychopathology, Reznick and colleagues developed the Retrospective Self-Report of Inhibition (RSRI) scale composed by 30 items that aimed to retrospectively assess behavioural inhibition during childhood (between the $1^{\text {st }}$ and $6^{\text {th }}$ school years). The aim of this manuscript is to confirm the RSRI psychometric properties in a Portuguese population and to investigate this instrument utility as a measure of behavioural inhibition during childhood. The first study, conducted with an adolescent sample $(n=296)$ with ages ranging between 12 and 18 years old, revealed that the RSRI shows a good internal consistency and a factorial structure similar to the original version. Evidence for the existence of two factors was maintained: fear/illness related to fears in general and somatic complaints; and a social/school factor that includes items related to fears in the school context and in several social situations. In the second study, conducted with a clinical and a control group (adolescents with a social anxiety disorder diagnosis $(n=76)$, other anxiety disorders $(n=28)$ and without psychopathology $(n=76)$ the scale and its dimensions were analysed regarding their discriminative power. Results show the bidirectional utility of the construct of behavioural inhibition, as assessed by the RSRI, showing that the social fears dimension presents a higher discriminative power when compared with the non-social fears dimension. Suggestions for future research include studies that reinforce these data and contribute to support the utility of this instrument for clinical research with Portuguese adolescents.

Keywords: RSRI scale, adolescence, self-report instruments, behavioural inhibition, social anxiety

\section{Introdução}

A inibição comportamental pode ser definida como uma predisposição temperamental das crianças para reagir consistentemente a acontecimentos novos e não familiares, tanto sociais como não-sociais, com moderação e 
constrangimento, juntamente com sinais de precaução e medo (Reznick, Hegeman, Kaufman, Woods, \& Jacobs, 1992). Estas crianças, quando enfrentam situações, pessoas ou objectos não familiares, respondem de forma particular caracterizada por uma tendência para manifestarem comportamentos passivos, de retraimento na exploração da situação e de procura da proximidade com a pessoa familiar. As crianças desinibidas mostram o padrão comportamental oposto. O conceito de inibição comportamental define, assim, as características temperamentais de insegurança com estranhos, cautelas excessivas em situações que envolvam risco de perigo e fracasso.

O comportamento inibido e desinibido tem sido visto como uma manifestação precoce da introversão e extroversão, respectivamente, nos adultos (Broberg, 1993; Broberg, Lamb, \& Hwang, 1990; Kagan, Reznick, \& Gibbons, 1989). Calcula-se que este temperamento de inibição comportamental esteja presente em cerca de 10 a $15 \%$ das crianças entre os 2 e os 3 anos de idade (Kagan, Reznick, \& Snidman, 1988). A relevância deste tipo de temperamento é confirmada pelo facto de ter sido observada uma considerável semelhança no padrão de inibição entre diferentes espécies (Mineka \& Zinbarg, 1995; Suomi, 1986), bem como entre diferentes culturas (Broberg, 1993; Broberg, et al., 1990; Kagan et al., 1994).

Em síntese, a investigação tem revelado que a inibição comportamental (IC) pode ser observada muito cedo na vida (Garcia-Coll, Kagan, \& Reznick, 1984) e parece ser estável ao longo do tempo (Reznick et al., 1986). Esta tendência comportamental parece estar sob um poderoso controlo genético (DiLalla, Kagan, \& Reznick, 1994) e está associada a manifestações fisiológicas específicas, tais como uma reactividade aumentada do sistema nervoso simpático (Kagan, 2001).

A inibição comportamental enquanto categoria de temperamento é, actualmente, um constructo largamente investigado, nomeadamente no âmbito da Psicologia Clínica, já que a evidência empírica tem sido pródiga em sugerir que uma história de inibição comportamental pode constituir um factor de risco para o desenvolvimento de perturbações ansiosas em geral e, em particular, da fobia social ou ansiedade social mal adaptativa. Torna-se assim imperativa a compreensão dos mecanismos através dos quais os factores de risco presentes na infância tendem a influenciar a expressão da psicopatologia ao longo da vida. Esta tarefa, não obstante a sua importância, não tem sido fácil, dados os anos e recursos necessários à condução de uma investigação longitudinal. Com o objectivo de restringir esta limitação, Reznick e colaboradores (1992) construíram um instrumento de auto-avaliação retrospectiva da inibição comportamental, tendo em vista obter uma medida da inibição comportamental expressa na infância (Reznick et al., 1992).

A escala de Auto-avaliação Retrospectiva da Inibição Comportamental (RSRI- Retrospective Self-Report of Inhibition) tem sido profusamente aplica- 
da na investigação sobre este tema e, especificamente, no estudo da relação entre a inibição comportamental e a ansiedade social (Biederman, et al., 2001; Cunha, 2005; Cunha, Soares, \& Pinto-Gouveia, 2008; Hayward, Killen, Kraemer, \& Taylor, 1998; Mick \& Telch, 1998; Neal, Edelmann, \& Glachan, 2002; Schwartz, Snidman, \& Kagan, 1999; Van Ameringen, Mancini, \& Oakman, 1998). Aplicada quer a adultos, quer a adolescentes, os seus resultados, de uma forma geral, tendem a confirmar esta associação (ibidem).

A escala permite obter uma medida da inibição comportamental manifesta na infância (entre o primeiro e o sexto ano de escolaridade) e é composta por questões centradas em comportamentos específicos que reflectem a definição teórica de inibição comportamental nesta faixa etária, isto é, um padrão persistente de constrangimento, precaução e evitamento em situações sociais e não sociais.

Procurando circunscrever as potenciais limitações decorrentes dos enviesamentos de memória, os autores elaboraram as questões da escala em torno de acontecimentos auto-biográficos específicos ou de estados emocionais com estes relacionados, em detrimento da utilização de perguntas que envolvessem inferências excessivas. Supõe-se que, desta forma, as recordações baseadas em acontecimentos específicos seriam mais precisas do que a lembrança de estados mais gerais.

Podem ser distinguidos dois factores independentes, designados por factor escola/situações sociais e factor medos/doenças, que têm revelado grande utilidade na investigação clínica, sugerindo, então, que a conceptualização bidimensional do constructo de inibição comportamental regista algum suporte empírico (Hayward et al., 1998; Neal et al., 2002; Van Ameringen et al., 1998). A investigação tem revelado que, relativamente à ansiedade social, a dimensão de medos sociais tem um maior poder discriminativo do que a dimensão de medos não sociais (ibidem).

Dada a inequívoca importância da avaliação da inibição comportamental na infância e necessidade de uma melhor compreensão do seu papel no desenvolvimento da psicopatologia, e tendo em conta as qualidades psicométricas evidenciadas pela RSRI, pareceu-nos oportuno adaptá-la para a língua portuguesa.

O objectivo deste artigo é confirmar as qualidades psicométricas da RSRI aplicada a uma população portuguesa e investigar a utilidade deste instrumento enquanto medida da inibição comportamental expressa na infância. O primeiro estudo, realizado numa amostra da comunidade, apresenta as características psicométricas e a análise da estrutura factorial deste instrumento. No segundo estudo, recorrendo a grupos clínicos e de controlo, procurou-se analisar a capacidade discriminativa da escala e suas dimensões, partindo da hipótese de que haveria uma associação específica com a perturbação de ansiedade social. 


\section{Método}

\section{Participantes}

Participaram 296 sujeitos, 140 do sexo masculino (47,3\%) e 156 do sexo feminino $(52,7 \%)$, com idades compreendidas entre os 12 e os 18 anos $(M=14,35 ; D P=1,91)$ e nível de escolaridade entre o $7^{\circ}$ e $12^{\circ}$ ano $(M=9,04$; $D P=1,72)$. Relativamente ao nível sócio-económico, distribuíram-se da seguinte forma: $33,6 \%$ apresentaram um nível sócio-económico baixo, $51,7 \%$ pertenciam ao nível médio e $32,1 \%$ ao nível alto. Não se verificaram diferenças significativas entre rapazes e raparigas relativamente à idade $(t=-1,82 ; p>0,50)$, anos de escolaridade $(t=-2,69 ; p>0,50)$, bem como na distribuição pelos níveis sócio-económicos $\left(\chi^{2}=1,29 ; p>0,50\right)$.

Esta amostra foi retirada de um estudo mais amplo realizado com uma amostra significativa de adolescentes $(N=2190)$, o qual procurou identificar a frequência e características da ansiedade social e possíveis factores de desenvolvimento (Cunha, 2005).

\section{Material}

A Escala de Auto-avaliação Retrospectiva da Inibição Comportamental (RSRI- Retrospective Self-Report of Inhibition, J. Steven Reznick, 1992) é formada por 30 questões que procuram avaliar, retrospectivamente, o grau de inibição comportamental do indivíduo durante a infância (entre o $1^{\circ}$ e o $6^{\circ}$ ano de escolaridade). $\mathrm{O}$ conteúdo das questões é baseado na perspectiva segundo a qual a inibição se define como uma tendência para a incerteza e a dúvida expressa muitas vezes, como medo e preocupação em situações sociais e não sociais.

Desta forma, os itens escolhidos procuram representar uma gama alargada de comportamentos associados à inibição comportamental, distribuídos por cinco domínios relevantes: (a) actividade do sistema nervoso simpático, com questões acerca de doenças, em geral, e queixas somáticas específicas, como alergias, obstipação e problemas de sono; (b) medo de coisas, situações e acontecimentos futuros, com questões acerca do medo do escuro, de animais, da escola, de actividades de grupo e de ser abandonado; (c) comportamentos destinados a diminuir os medos, com questões à volta da utilização de luz de presença ou de objectos transaccionais para adormecer; (d) comportamentos que reflectem o medo do desconhecido como, as questões acerca de pesadelos, de provar sabores novos e de ficar receoso na escola; (e) comportamentos que revelam aptidões sociais pobres como, perguntas sobre as brincadeiras na escola, o comportamento nas festas, a popularidade, as actividades extra-curriculares e a interacção com os pares. 
Cada uma das questões é respondida com base numa escala de tipo Likert de 5 pontos ('nunca', 'poucas vezes', 'às vezes', 'muitas vezes' e 'sempre') ou, em determinadas questões, são utilizados termos apropriados e comparáveis. As respostas ao item variam do comportamento menos inibido para o mais inibido e, quanto maior é a pontuação, maior é a inibição comportamental assim medida.

Estudos desenvolvidos por Reznick e colaboradores na aplicação desta escala revelaram que esta possui boas características psicométricas (Reznick et al., 1992) Os resultados evidenciaram uma moderada consistência interna, com o valor de alfa de Cronbach, para as 30 questões, igual a 0,79. A análise factorial revelou a existência de dois factores distintos: o primeiro, designado escola/situações sociais, engloba os itens relacionados com medos em contexto escolar e em diversas situações sociais (e.g. "Ficavas nervoso quando te chamavam ao quadro?"; "Quando ias a festas gostavas de participar em jogos?"); o segundo factor, medos/doenças, diz respeito a situações relacionadas com medos e perturbações físicas (e.g. "Tinhas medo do escuro?"; "Tiveste doenças ou sintomas como dores de cabeça ou dores de barriga, para as quais os médicos não foram capazes de encontrar uma causa?". A validade da RSRI foi estudada através da comparação dos resultados obtidos nos questionários preenchidos pelos jovens e pelos pais, isto é, dados da inibição comportamental avaliada pelo próprio e pelos pais, revelando uma forte associação positiva entre estas duas medidas (Reznick et al., 1992).

\section{Procedimento}

Os itens da Retrospective Self-Report of Inhibition foram traduzidos tomando em consideração a equivalência lexical e gramatical, a equivalência conceptual e, finalmente, o emparelhamento do conteúdo do item com a dimensão que se propõe avaliar. Adoptando as sugestões e indicações recomendadas, foi feita a tradução, seguida de retroversão e revisto este procedimento por um tradutor especializado a fim de assegurar a equivalência de conteúdo das versões americana e portuguesa (de Figueiredo, 1980). Comparativamente à escala original, foi feita uma alteração relativamente às formas verbais, passando-as para a segunda pessoa no sentido de melhor se ajustar à população alvo a utilizar, nomeadamente adolescentes. Prosseguiu-se com a administração da escala a pequenos grupos (da comunidade e clínica) com o objectivo de verificar a acessibilidade do vocabulário e a compreensão unívoca dos itens. ${ }^{4} \mathrm{O}$ último passo consistiu na aplicação desta escala a várias dezenas de jovens do distrito de Coimbra, na própria sala de aulas, durante o

\footnotetext{
${ }^{4}$ Utilizando estes procedimentos, verificou-se igualmente um aspecto da validade facial do instrumento.
} 
período escolar, juntamente com outros instrumentos de medida e uma entrevista clínica que faziam parte de uma investigação alargada sobre ansiedade social na adolescência (Cunha, 2005).

\section{Resultados}

\section{Estudo dos itens da escala}

As médias, desvios-padrão e correlações item-total para cada item são apresentados no Quadro 1.

Quadro 1: Estudo dos itens da Escala-Médias, desvios-padrão e correlações item-total para cada item e para o total da escala

\begin{tabular}{|c|c|c|c|}
\hline \multirow[b]{2}{*}{ Itens } & \multicolumn{3}{|c|}{ Item-Total } \\
\hline & $\mathbf{M}$ & DP & $\mathbf{r}$ \\
\hline $\begin{array}{l}\text { 1. Em média, quantas vezes por ano faltaste às aulas por } \\
\text { motivo de doença? }\end{array}$ & 1,59 & 0,90 & 0,17 \\
\hline $\begin{array}{l}\text { 2. Em média, quantas vezes por ano te mandaram ao } \\
\text { gabinete de enfermagem por motivo de doença? }\end{array}$ & 1,10 & 0,39 & 0,08 \\
\hline $\begin{array}{l}\text { 3. Tiveste doenças ou sintomas como dores de cabeça ou } \\
\text { dores de barriga, para as quais os médicos não foram } \\
\text { capazes de encontrar uma causa? }\end{array}$ & 1,39 & 0,74 & 0,29 \\
\hline 4. Com que frequência tinhas pesadelos? & 2,42 & 1,04 & 0,36 \\
\hline 5. Tinhas medo do escuro? & 2,07 & 1,22 & 0,39 \\
\hline $\begin{array}{l}\text { 6. Era necessário os teus pais, ou tu, verificarem que não } \\
\text { havia nada debaixo da cama ou dentro do armário antes } \\
\text { de te deitares? }\end{array}$ & 1,31 & 0,74 & 0,41 \\
\hline $\begin{array}{l}\text { 7. Precisavas de ter um peluche, um cobertor, ou um } \\
\text { brinquedo especial contigo para conseguir adormecer? }\end{array}$ & 1,78 & 1,26 & 0,30 \\
\hline $\begin{array}{l}\text { 8. Tinhas medo de cães, gatos ou outros animais domés- } \\
\text { ticos? }\end{array}$ & 1,48 & 0,93 & 0,24 \\
\hline $\begin{array}{l}\text { 9. Tinhas medo de animais desconhecidos, como os que } \\
\text { encontravas na rua ou em casa de outras pessoas? }\end{array}$ & 2,06 & 1,11 & 0,34 \\
\hline $\begin{array}{l}\text { 10. Tinhas medo de ser raptado, ou de ser separado dos } \\
\text { teus pais? }\end{array}$ & 2,36 & 1,28 & 0,45 \\
\hline $\begin{array}{l}\text { 11. Incomodava-te quando os teus pais te deixavam com } \\
\text { uma pessoa nova para tomar conta de ti? }\end{array}$ & 1,89 & 1,07 & 0,45 \\
\hline $\begin{array}{l}\text { 12. Quando os teus pais saíam sem ti, tinhas medo de } \\
\text { que eles pudessem não voltar?) }\end{array}$ & 1,78 & 1,14 & 0,44 \\
\hline 13. Costumavas dormir em casa dos teus amigos? & 3,79 & 1,00 & 0,18 \\
\hline 14. Experimentavas novos pratos? & 2,38 & 0,97 & 0,17 \\
\hline $\begin{array}{l}\text { 15. Costumavas ficar com medo no primeiro dia do novo } \\
\text { ano lectivo? }\end{array}$ & 1,91 & 0,98 & 0,62 \\
\hline
\end{tabular}




\begin{tabular}{|c|c|c|c|}
\hline $\begin{array}{l}\text { 16. Alguma vez fingiste estar doente para evitar ir às } \\
\text { aulas ou a acontecimentos sociais? }\end{array}$ & 1,32 & 0,67 & 0,24 \\
\hline 17. Ficavas nervoso quando te chamavam ao quadro? & 2,06 & 1,05 & 0,58 \\
\hline $\begin{array}{l}\text { 18. Ficavas nervoso quando o professor te fazia pergun- } \\
\text { tas nas aulas, mesmo se soubesses a resposta? }\end{array}$ & 1,83 & 1,00 & 0,63 \\
\hline $\begin{array}{l}\text { 19. Os teus professores tinham dificuldades em ouvir-te } \\
\text { quando falavas ou respondias às perguntas nas aulas? }\end{array}$ & 1,83 & 1,00 & 0,54 \\
\hline $\begin{array}{l}\text { 20. Se não percebesses alguma coisa na aula, pedias } \\
\text { ajuda ao professor? }\end{array}$ & 2,75 & 1,19 & 0,47 \\
\hline $\begin{array}{l}\text { 21. Durante o intervalo, brincavas com o grupo de alu- } \\
\text { nos dominante? }\end{array}$ & 2,52 & 1,14 & 0.33 \\
\hline 22. Quando ias a festas gostavas de participar em jogos? & 2,26 & 1,15 & 0,41 \\
\hline 23. Gostavas de conhecer outras crianças da tua idade? & 1,98 & 0,96 & 0,43 \\
\hline $\begin{array}{l}\text { 24. A tua voz tornava-se esganiçada, tremida ou sumida } \\
\text { quando falavas perante um grupo de pessoas? }\end{array}$ & 1,96 & 1,05 & 0,56 \\
\hline 25. Quão popular te sentias? & 3,09 & 1,04 & 0,34 \\
\hline $\begin{array}{l}\text { 26. Alguma vez tiveste problemas ou tiveste de ir ao } \\
\text { médico por causa de alergias, insónias ou obstipação? }\end{array}$ & 1,69 & 0,94 & 0,13 \\
\hline $\begin{array}{l}\text { 27. Precisavas de uma luz de presença ou uma luz acesa } \\
\text { no corredor para adormecer? }\end{array}$ & 1,76 & 1,21 & 0,27 \\
\hline $\begin{array}{l}\text { 28. Participavas com vontade em grupos de música ou } \\
\text { de teatro? }\end{array}$ & 3,02 & 1,33 & 0,25 \\
\hline 29. Sentias-te facilmente magoado? & 2,34 & 1,08 & 0,54 \\
\hline $\begin{array}{l}\text { 30. Dizias alguma coisa aos amigos ou aos familiares } \\
\text { quando te zangavas com eles? }\end{array}$ & 3,17 & 1,14 & 0,25 \\
\hline TOTAL & 2,09 & 0,45 & \\
\hline
\end{tabular}

A análise das correlações item-total indica que 7 dos itens da escala possuem correlações item-total inferiores a 0.25 . Tratam-se do item 1 ("Em média, quantas vezes por ano faltaste às aulas por motivo de doença?"), item 2 ("Em média, quantas vezes por ano te mandaram ao gabinete de enfermagem por motivo de doença?"), item 8 ("Tinhas medo de cães, gatos ou outros animais domésticos?"), item 13 ("Costumavas dormir em casa dos teus amigos?"), item 14 ("Experimentavas novos pratos?), item 16 ("Alguma vez fingiste estar doente para evitar ir às aulas ou a acontecimentos sociais?") e item 26 ("Alguma vez tiveste problemas ou tiveste de ir ao médico por causa de alergias, insónias ou obstipação?”).

Estas correlações são baixas, mas os seus valores continuam a ser estatisticamente significativos $(p<0.01)$, sendo a média da correlação item total superior 0.30 . Por outro lado, há ainda a acrescentar que a eliminação destes itens não aumentava a consistência interna da escala. Por estes motivos, e após a análise factorial da escala, discutida de seguida, os itens acima descritos foram mantidos. 


\section{Análise Factorial}

Com o objectivo de determinar a estrutura factorial desta escala recorreu-se ao método de análise de componentes principais seguido de rotação Varimax (efectuada com o programa SPSS versão14.0 para Windows). Para a medida de Kaiser-Meyer-Olkin (KMO) foi obtido o valor de 0.83 , revelando uma boa adequação da amostragem, e foi significativo o teste de esfericidade de Bartllet $\left(\chi^{2}=2306.39 ; p<0.000\right)$, demonstrando que a matriz de intercorrelações é significativamente diferente de uma matriz de identidade.

Numa primeira análise factorial exploratória, os resultados revelaram nove factores com valores próprios superiores a 1 (eingenvalues) que explicavam $58.70 \%$ da variância total. Procedeu-se seguidamente a uma rotação Varimax dos eixos para melhor leitura da estrutura factorial, recorrendo, para tal, a um procedimento semelhante ao do estudo original, seleccionando dois factores. Esta solução de dois factores explica 28.14\% (contra $25.33 \%$ da versão original). A ordem dos factores surge invertida no presente estudo em comparação com a versão original. No presente estudo o primeiro factor da solução é constituído pelos itens relativos a medos e doenças e o segundo factor diz respeito a itens relacionados com a escola e situações sociais. No Quadro 2 são apresentados os factores com os itens que os compõem e respectivas saturações, as comunalidades, assim como os valores de consistência interna de cada factor.

Quadro 2: Factores, comunalidades, alpha de Cronbach de cada factor e saturações dos itens que os compõem

\begin{tabular}{|c|c|c|c|}
\hline & Factor 1 & Factor 2 & $\begin{array}{c}\text { Comuna- } \\
\text {-lidade }\end{array}$ \\
\hline Itens da escala & 0,71 & $-0,11$ & 0,66 \\
\hline Factor $1-$ Medos/Doenças $(\alpha=0,77)$ & $\mathbf{0 , 6 1}$ & 0,04 & 0,51 \\
\hline Tinhas medo do escuro? (5) & $\mathbf{0 , 5 4}$ & 0,18 & 0,58 \\
\hline $\begin{array}{l}\text { Era necessário os teus pais, ou tu, verificarem } \\
\text { que não havia nada debaixo da cama ou dentro } \\
\text { do armário antes de te deitares? (6) }\end{array}$ & $\mathbf{0 , 5 4}$ & 0,47 & 0,61 \\
\hline $\begin{array}{l}\text { Incomodava-te quando os teus pais te deixavam } \\
\text { com uma pessoa nova para tomar conta de ti? } \\
\text { (11) }\end{array}$ & $\mathbf{0 , 5 3}$ & 0,51 & 0,71 \\
\hline $\begin{array}{l}\text { Costumavas ficar com medo no primeiro dia do } \\
\text { novo ano lectivo? (15) }\end{array}$ & $\mathbf{0 , 5 3}$ & $-0,05$ & 0,46 \\
\hline $\begin{array}{l}\text { Ficavas nervoso quando o professor te fazia } \\
\text { perguntas nas aulas, mesmo se soubesses a } \\
\text { resposta? (18) }\end{array}$ & $\mathbf{0 , 5 2}$ & 0,37 & 0,47 \\
\hline
\end{tabular}




\begin{tabular}{|c|c|c|c|}
\hline $\begin{array}{l}\text { Precisavas de ter um peluche, um cobertor, ou } \\
\text { um brinquedo especial contigo para conseguir } \\
\text { adormecer? ( } 7 \text { ) }\end{array}$ & $\mathbf{0 , 5 1}$ & $-0,12$ & 0,60 \\
\hline Sentias-te facilmente magoado? (29) & $\mathbf{0 , 5 1}$ & 0,05 & 0,42 \\
\hline $\begin{array}{l}\text { Precisavas de uma luz de presença ou uma luz } \\
\text { acesa no corredor para adormecer? (27) }\end{array}$ & $\mathbf{0 , 5 0}$ & 0,18 & 0,68 \\
\hline Com que frequência tinhas pesadelos? (4) & 0,46 & 0,27 & 0,67 \\
\hline $\begin{array}{l}\text { Tinhas medo de ser raptado, ou de ser separado } \\
\text { dos teus pais? (10) }\end{array}$ & $\mathbf{0 , 4 3}$ & 0,10 & 0,78 \\
\hline $\begin{array}{l}\text { Quando os teus pais saíam sem ti, tinhas medo } \\
\text { de que eles pudessem não voltar? (12) }\end{array}$ & $\mathbf{0 , 3 9}$ & 0,09 & 0,55 \\
\hline $\begin{array}{l}\text { Tinhas medo de animais desconhecidos, como } \\
\text { os que encontravas na rua ou em casa de outras } \\
\text { pessoas? (9) }\end{array}$ & & & \\
\hline $\begin{array}{l}\text { Tiveste doenças ou sintomas como dores de } \\
\text { cabeça ou dores de barriga, para as quais os } \\
\text { médicos não foram capazes de encontrar uma } \\
\text { causa? (3) }\end{array}$ & 0,05 & $\mathbf{0 , 6 7}$ & 0,61 \\
\hline Factor 2 - Escola/Situações Sociais $(\alpha=0,78)$ & 0,06 & 0,66 & 0,67 \\
\hline $\begin{array}{l}\text { Quando ias a festas gostavas de participar em } \\
\text { jogos? (22) }\end{array}$ & 0,33 & $\mathbf{0 , 6 0}$ & \\
\hline $\begin{array}{l}\text { Gostavas de conhecer outras crianças da tua } \\
\text { idade? (23) }\end{array}$ & 0,23 & $\mathbf{0 , 5 7}$ & 0,48 \\
\hline $\begin{array}{l}\text { A tua voz tornava-se esganiçada, tremida ou } \\
\text { sumida quando falavas perante um grupo de } \\
\text { pessoas? (24) }\end{array}$ & $-0,12$ & $\mathbf{0 , 5 6}$ & 0,51 \\
\hline $\begin{array}{l}\text { Se não percebesses alguma coisa na aula, } \\
\text { pedias ajuda ao professor? (20) }\end{array}$ & 0,06 & $\mathbf{0 , 5 2}$ & 0,62 \\
\hline $\begin{array}{l}\text { Participavas com vontade em grupos de música } \\
\text { ou de teatro? (28) }\end{array}$ & 0,41 & $\mathbf{0 , 5 1}$ & 0,71 \\
\hline $\begin{array}{l}\text { Durante o intervalo, brincavas com o grupo de } \\
\text { alunos dominante? }(21)\end{array}$ & 0,10 & 0,48 & 0,53 \\
\hline $\begin{array}{l}\text { Ficavas nervoso quando te chamavam ao qua- } \\
\text { dro? (17) }\end{array}$ & 0,33 & 0,46 & 0,53 \\
\hline Quão popular te sentias? (25) & 0,01 & $\mathbf{0 , 3 8}$ & 0,45 \\
\hline \multicolumn{4}{|l|}{$\begin{array}{l}\text { Os teus professores tinham dificuldades em } \\
\text { ouvir-te quando falavas ou respondias às per- } \\
\text { guntas nas aulas? (19) }\end{array}$} \\
\hline $\begin{array}{l}\text { Dizias alguma coisa aos amigos ou aos familia- } \\
\text { res quando te zangavas com eles? (30) }\end{array}$ & 0,28 & 0,08 & 0,82 \\
\hline $\begin{array}{l}\text { Itens que não saturaram significativamente em } \\
\text { qualquer factor }\end{array}$ & 0,27 & 0,13 & 0,62 \\
\hline $\begin{array}{l}\text { Tinhas medo de cães, gatos ou outros animais } \\
\text { domésticos? (8) }\end{array}$ & 0,20 & $-0,08$ & 0,62 \\
\hline
\end{tabular}




\begin{tabular}{lccc}
\hline $\begin{array}{l}\text { Alguma vez fingiste estar doente para evitar ir } \\
\text { às aulas ou a acontecimentos sociais? (16) }\end{array}$ & 0,16 & 0,12 & 0,56 \\
$\begin{array}{l}\text { Em média, quantas vezes por ano te mandaram } \\
\text { ao gabinete de enfermagem por motivo de } \\
\text { doença? (2) }\end{array}$ & 0,12 & 0,06 & 0,61 \\
$\begin{array}{l}\text { Em média, quantas vezes por ano faltaste às } \\
\text { aulas por motivo de doença? (1) }\end{array}$ & $-0,01$ & 0,29 & 0,43 \\
$\begin{array}{l}\text { Alguma vez tiveste problemas ou tiveste de ir } \\
\text { ao médico por causa de alergias, insónias ou } \\
\text { obstipação? (26) }\end{array}$ & 0,14 & 0,16 & 0,66 \\
$\begin{array}{l}\text { Experimentavas novos pratos? (14) } \\
\begin{array}{l}\text { Costumavas dormir em casa dos teus amigos? } \\
\text { (13) }\end{array}\end{array}$ & & \\
\hline
\end{tabular}

Na determinação da composição dos factores tivemos em conta a sugestão de Floyd e Widaman (Floyd \& Widaman, 1995), retendo os itens cuja saturação no factor fosse superior ou igual a 0,35 , bem como os itens que, embora saturando significativamente em dois factores, apresentassem uma diferença entre as respectivas saturações superior a .30. Este último critério permitia reter itens que tivessem uma saturação alta num factor e que apresentassem marginalmente uma saturação significativa num outro factor, tendo este procedimento sido aplicado apenas a dois itens da escala (itens 17 e 19). Por fim, nesta análise, todos os itens não seleccionados eram, também, aqueles que na análise correlação item-total apresentavam valores de correlações mais baixos (itens, $1,2,8,13,14,16$ e $26 ; r<0,25$ ).

Os factores encontrados para a escala replicam, quase inteiramente, a estrutura factorial obtida no estudo original do instrumento. Uma análise mais detalhada na comparação das estruturas factoriais das duas versões desta escala - versão americana e portuguesa - permite-nos verificar que, apesar da semelhança e concordância encontradas, existem também pequenas diferenças quanto à composição dos factores, nomeadamente no que diz respeito à distribuição dos itens $1,2,16$ e 26 .

Como se pode ver no Quadro 3, os itens 1, 2, 16 e 26 na versão americana são incluídos no factor relacionado com medos e doenças, enquanto no nosso estudo estes itens não saturaram significativamente em qualquer factor $(r<0,25)$, o que é consistente com o facto de apresentarem valores baixos de correlação item-total $(r<0,25)$. Por sua vez, os itens 11 e 29 , que não eram incluídos originalmente, apresentaram valores de saturação significativos neste factor. Por último, na versão portuguesa os itens 15 e 18 saturam significativamente nos dois factores e não apenas em um, como na versão original. 
Quadro 3: Comparação das versões americana e portuguesa da RSRI relativamente aos itens que constituem cada um dos factores da escala

\begin{tabular}{lll}
\hline RSRI/ Factores & $\begin{array}{l}\text { Versão Americana } \\
\text { Itens }\end{array}$ & $\begin{array}{l}\text { Versão Portuguesa } \\
\text { Itens }\end{array}$ \\
\hline Medos/Doenças & $\mathbf{1 , 2}, 3,4,5,6,7,10,12, \mathbf{1 6}, \mathbf{2 6}$, & $3,4,5,6,7, \mathbf{9}, 10, \mathbf{1 1}, 12,27, \mathbf{2 9}$ \\
& 27 & \\
Escola/Sit. Sociais & $\mathbf{1 5}, 17, \mathbf{1 8}, 19,20,21,22,23$, & $17,19,20,21,22,23,24,25,28$, \\
& $24,25,28,30$ & 30 \\
\hline
\end{tabular}

Para avaliar a correspondência entre estes factores e os factores obtidos por Reznick e colaboradores na população americana, calcularam-se as correlações entre as estruturas factoriais obtidas nos dois países. Por outras palavras, utilizando-se a amostra deste estudo, calcularam-se as correlações de Pearson entre cada escala derivada da nossa análise factorial e a escala correspondente da versão americana. Como seria de esperar, obteve-se uma acentuada correspondência entre os factores portugueses e americanos $(r=0,93$ para o factor 1-Medo s/Doenças; $r=0,98$ para o Factor 2-Escola/Situações Sociais $(p<0,001)$.

Em resumo, pode dizer-se que o padrão de distribuição dos itens na versão Portuguesa da RSRI aqui apresentado é idêntico ao da versão americana, ou seja, que há uma equivalência conceptual entre as duas versões.

No que diz respeito ao estudo das intercorrelações ente os factores e o total da escala, conclui-se que representam dimensões relativamente independentes, tendo-se obtido valores de correlação de 0,82 para cada um dos factores com o total da escala e de 0,41 para o valor de correlação entre os dois factores $(p<0,001)$.

\section{Consistência interna}

A consistência interna de uma escala é uma das características que garante que os itens são da mesma família ou que avaliam um mesmo constructo. Para analisar a consistência interna, recorremos ao alfa de Cronbach. Os resultados indicaram para os 30 itens da escala um valor de $0,84(0,79$ na versão original) o que revela uma boa consistência interna. A magnitude dos valores é boa e semelhante à versão original, sendo o valor mediano da correlação item-total igual 0,35 ( $r=0,31$ na versão original). 


\section{Valores descritivos}

O Quadro 4, onde são apresentados as médias e os desvios-padrão para cada um dos factores e total do instrumento em função do género e idade dos participantes, permite uma análise mais detalhada dos resultados obtidos na escala de Auto-avaliação Retrospectiva da Inibição Comportamental (RSRI).

Quadro 4: Médias e desvios-padrão dos factores e total da escala segundo o género e o grupo de idade

\begin{tabular}{|c|c|c|c|c|c|c|}
\hline & \multicolumn{6}{|c|}{ RSRI } \\
\hline & \multicolumn{2}{|c|}{ F1-Medos/doenças } & \multicolumn{2}{|c|}{ F2 -Escola/Sit. Sociais } & \multicolumn{2}{|c|}{ Total } \\
\hline & $M$ & DP & $M$ & DP & $M$ & DP \\
\hline \multicolumn{7}{|l|}{ Género } \\
\hline Masculino $(n=140)$ & 19,22 & 5,48 & 23,51 & 5,76 & 59,45 & 11,48 \\
\hline Feminino $(n=156)$ & 22,91 & 6,97 & 26,67 & 6,73 & 60,02 & 14,16 \\
\hline$t(294)$ & \multicolumn{2}{|c|}{$-5,02 * * *$} & \multicolumn{2}{|c|}{$-2,95^{* *}$} & \multicolumn{2}{|c|}{$-4,35 * * *$} \\
\hline \multicolumn{7}{|l|}{ Idade } \\
\hline $12-15(n=201)$ & 20,63 & 6,16 & 24,30 & 6,11 & 62,03 & 12,64 \\
\hline $16-18(\mathrm{n}=95)$ & 22,28 & 7,25 & 25,36 & 6,86 & 67,77 & 14,63 \\
\hline$t(294)$ & \multicolumn{2}{|c|}{$-2,02 *$} & \multicolumn{2}{|c|}{$-1,34$} & \multicolumn{2}{|c|}{$-1,64$} \\
\hline
\end{tabular}

Nota: $* * * p<0,001 ; * * p<0,005 ; * p<0,05$

Contrariamente aos resultados obtidos na escala original para adultos, nos quais não se verificaram diferenças significativas no género, neste estudo as raparigas obtêm valores significativamente mais elevados do que os rapazes, quer no total da escala $t(294)=-4,35 ; p<0,001$, quer em ambos os factores $[(t(294)=-5,02 ; p<0,001$ para o factor Medos /doenças; $t(294)=$ $-2,95 ; p<0,005$ para o factor Escola/Situações Sociais].

Relativamente à idade, verifica-se que apenas no factor medos/doenças existe uma diferença pequena $(t(294)=-2,02)$, mas ainda estatisticamente significativa $(p=0,04)$, entre os valores médios obtidos nos grupos de idade referenciados, com os mais novos a revelarem mais medos e mais queixas somáticas do que os mais velhos.

Quando comparados os valores médios obtidos no total e nos factores da escala em função do nível escolar (ensino básico versus ensino secundário) e nível sócio-económico (alto, médio e baixo) não se registaram diferenças significativas. 


\section{Estudo 2 - Validade discriminativa}

Dada a ligação apontada pela literatura científica entre a inibição comportamental e as perturbações ansiosas, em geral, e a perturbação de ansiedade social, em especial, este estudo visou identificar a capacidade discriminativa da inibição comportamental medida pela RSRI, recorrendo à comparação entre jovens com uma fobia social, jovens com outras perturbações ansiosas e jovens sem qualquer diagnóstico clínico.

\section{Participantes}

Com base nos diagnósticos obtidos numa entrevista clínica (Anxiety Disorders Interview Schedule for Children: ADIS-C), foram constituídos três grupos de comparação. O grupo de indivíduos com fobia social (FS) é formado por 76 jovens, 27 rapazes e 49 raparigas, com uma média de idades de $14.61(D P=1.93)$. O grupo de controlo 'normais' $(\mathbf{C N})$, seleccionado à semelhança do grupo de fóbicos sociais, é constituído por 76 jovens, 27 rapazes e 49 raparigas, com uma média de idade de 14.66 anos $(D P=1.93)$. Por último, o grupo de outras perturbações ansiosas (OPA) é formado por 28 indivíduos, 11 rapazes e 17 raparigas, sendo a média de idades de 14.32 $(D P=2.13)$. As perturbações ansiosas deste grupo incluem a fobia específica (19), perturbação de ansiedade generalizada (4), perturbação obsessivo-compulsiva (3) perturbação de ansiedade de separação (1) e perturbação de pós-stress traumático (1).

No seu conjunto, os três grupos não diferem significativamente, do ponto de vista estatístico, na idade $[F(2,177)=0,290 ; p>0,05]$ ou nos anos de escolaridade $[F(2,177)=0,811 ; p>0,05]$. Os três grupos são ainda semelhantes quanto à proporção de rapazes e raparigas $\left(\chi^{2}=0,145 ; p>0,05\right)$, bem como relativamente à distribuição do nível sócio-económico $\left(\chi^{2}=5,765 ; p>0,05\right)$.

\section{Instrumentos}

Para além da RSRI, descrita no estudo 1, foram utilizados outros instrumentos de auto-resposta na avaliação de variáveis psicossociais (estilos parentais, estilos de vinculação, comparação social) e uma entrevista clínica estruturada (ADIS-IV for children; Silverman \& Albano, 1996) para o estabelecimento de diagnósticos clínicos e para a identificação de um grupo de controlo sem patologia.

A Entrevista Estruturada para as Perturbações de Ansiedade na Infância e Adolescência - ADIS-IV for children - (Silverman \& Albano, 1996) permite o diagnóstico das perturbações de ansiedade, das perturbações afec- 
tivas (Distimia e Perturbação Depressiva Major), bem como da Perturbação de Défice de Atenção e Hiperactividade (Silverman \& Albano, 1996). Em relação a cada uma destas categorias, são avaliados os aspectos clinicamente relevantes, tais como aspectos situacionais e cognitivos que geram ansiedade, intensidade da ansiedade, grau de evitamento, duração das dificuldades sentidas e acontecimentos precipitantes.

\section{Procedimento}

A entrevista foi conduzida individualmente por um psicólogo com treino prévio na utilização da entrevista de diagnóstico. Todas as entrevistas foram apresentadas e discutidas em reuniões semanais com a equipa de investigadores constituída por psicólogos ligados ao CINEIC (Centro de Investigação do Núcleo de Estudos e Intervenções Cognitivo-Comportamentais) da Faculdade de Psicologia e Ciências da Educação e à consulta de Psicoterapia Cognitivo-comportamental dos Hospitais da Universidade de Coimbra.

\section{Resultados}

Neste estudo estávamos interessados em investigar em que medida a inibição comportamental e as suas dimensões específicas (medos sociais e não-sociais) discriminavam a fobia social, enquanto perturbação ansiosa específica. Como hipótese de trabalho, partimos do pressuposto que os jovens com fobia social se distinguiriam dos jovens normais nos padrões de inibição na infância evidenciando, os primeiros, valores mais elevados.

Uma vez que a variável género se mostrou relacionada com a variável dependente (inibição comportamental, avaliada pela medida global da RSRI), realizámos ANOVAS com dois factores fixos para analisar a relação entre a inibição comportamental e a fobia social e a possível influência do género nesta relação $0^{5}$. Utilizámos os três grupos daatrás descritos e o género dos participantes como factores fixos, e o total e cada um dos factores da RSRI como variáveis dependentes, analisadas separadamente.

Os resultados dos testes univariados mostram que os grupos diferem em termos da medida global de inibição na infância $[F(2,174)=28,42$;

\footnotetext{
${ }^{5}$ Optámos pela Anova com dois factores fixos em relação à monofactorial (One-way,) uma vez que o segundo factor (género) se mostrou relacionado com a variável dependente, permitindo, assim, a quantificação dos efeitos isolados dos dois factores (grupos de comparação e género), diminuindo o erro do modelo. Em segundo lugar, este tipo de análise permitiria identificar e quantificar, caso existisse, a relação de interacção entre os dois factores, cujo efeito não poderia ser explicado por cada factor isoladamente. Por último, por permitir generalizar o estudo, caso não exista interacção entre os dois factores.
} 
$p<0,001]$, bem como relativamente aos medos $e$ doenças $[F(2,174)=17,20$; $p<0,001]$ e em relação à inibição na escola e em situações sociais $[F(2$, $174)=16,96, p<0,001]$, avaliados pelos factores da escala. Em relação ao efeito do género, verifica-se, nesta amostra, que os rapazes e raparigas diferem entre si na forma como auto-avaliam a inibição comportamental $[F(1$, $174)=4,41, p<0,050]$. Não se verifica qualquer interacção género $\mathrm{x}$ grupos de comparação para qualquer das variáveis estudadas $(p>0,050)$.

A realização de testes post-hoc (Tukey) permitiu localizar as diferenças entre os grupos (Quadro 5), verificando-se que o grupo de participantes com uma fobia social apresenta valores significativamente mais altos do que os normais $(p<0,001)$ e do que os jovens com outras perturbações ansiosas $(p<0,050)$, quer no total da RSRI, quer no factor de inibição relacionada com a escola ou situações sociais ( $p<0,001$ para as diferenças entre os grupos).

Em relação ao factor medos e doenças, os resultados mostram que os fóbicos sociais apenas diferem dos normais por apresentarem valores significativamente mais altos $(p<0,001)$, não se diferenciando dos jovens com outras perturbações ansiosas $(p>0,050)$.

Quadro 5: Médias, desvios-padrão e testes de comparações múltiplas em função dos grupos clínicos para as medidas de inibição comportamental (RSRI-total e factores)

\begin{tabular}{|c|c|c|c|c|c|c|c|c|c|}
\hline & \multicolumn{2}{|c|}{$\begin{array}{c}\text { Normais } \\
(N=76)\end{array}$} & \multicolumn{2}{|c|}{$\begin{array}{c}\text { Fobia Social } \\
\qquad(N=76)\end{array}$} & \multicolumn{2}{|c|}{$\begin{array}{c}\text { Out.Pertur } \\
\text { bações } \\
\text { Ansiosas } \\
(\mathbf{N}=\mathbf{2 8})\end{array}$} & \multirow[b]{2}{*}{$F$} & \multirow[b]{2}{*}{$p$} & \multirow[b]{2}{*}{ Post-hoc } \\
\hline & $\mathbf{M}$ & DP & $\mathbf{M}$ & DP & M & DP & & & \\
\hline RSRI - total & 57,82 & 11,08 & 74,01 & 14,22 & 64,04 & 10,74 & 32,37 & .000 & $\mathrm{FS}>\mathrm{CN}^{* *} ; \mathrm{FS}>\mathrm{OPA}^{*}$ \\
\hline Medos/doenças & 19,20 & 5,70 & 25,83 & 7,23 & 22,61 & 5,14 & 20,91 & .000 & $\mathrm{FS}>\mathrm{CN}^{* *} ; \mathrm{OPA}>\mathrm{CN}^{*}$ \\
\hline Escola/sit. sociais & 22,86 & 5,99 & 28,82 & 6,44 & 24,68 & 5.96 & 18,06 & .000 & $\mathrm{FS}>\mathrm{CN}^{* *} ; \mathrm{FS}>\mathrm{OPA}^{* *}$ \\
\hline
\end{tabular}

Nota: ${ }^{* *} p<0,001 ;{ }^{*} p<0,05$

Por sua vez, o grupo com outras perturbações ansiosas, para além das diferenças apontadas em relação ao grupo de fobia social, apenas se diferencia do grupo de jovens sem patologia no que respeita ao factor medos e doenças, revelando valores estatisticamente mais elevados do que estes $(\mathrm{p}<0,050)$.

\section{Discussão}

Dada a utilidade da avaliação da inibição comportamental através de um instrumento retrospectivo de auto-resposta, este estudo adaptou a RSRI à 
população adolescente portuguesa, utilizando uma amostra de adolescentes. Para o efeito, para além dos devidos procedimentos de tradução e adaptação para a língua Portuguesa, foram feitas pequenas modificações no sentido de melhorar a aplicação da Escala a jovens adolescentes.

Seguindo de perto os procedimentos dos autores da escala original, verificou-se que a escala portuguesa possui uma boa consistência interna e uma estrutura factorial idêntica à escala original para adultos, tendo sido igualmente identificados dois factores relativos a medos $e$ doenças (medos não sociais) e relativos a medos relacionados com a escola e situações sociais (medos sociais).

$\mathrm{Na}$ análise factorial da escala, os 7 itens que não foram seleccionados foram também aqueles que na análise de correlação item-total apresentavam valores de correlação mais baixos (inferiores a 0,25). Os itens $1,2,16$ e 26 reportam-se à frequência de doenças ou queixas somáticas, enquanto os itens 8, 13 e 14 referem-se a medos de animais ou de experiências novas (e.g., dormir em casa de amigos ou experimentar sabores novos), sugerindo que estes conteúdos não estão relacionados de uma forma tão robusta com o constructo medido.

Em relação ao destino destes sete itens excluídos da análise factorial, há duas opções. A mais simples seria a de retirar os itens da escala. Contudo, julgamos este procedimento precoce e pensamos que se deve aguardar por novos estudos com outras populações para tomar uma decisão mais definitiva. No entanto, no que toca ao item 2 - "Em média, quantas vezes por ano te mandaram ao gabinete de enfermagem por motivo de doença?" - julgamos que ele não se adequa ao contexto português, uma vez que, ao contrário do que acontece nas escolas norte-americanas, país de origem do instrumento, os estabelecimentos de ensino em Portugal não estão equipados, na sua maioria, com gabinetes de enfermagem. Assim sendo, futuramente este item deve ser retirado.

Tendo em conta estes dados, aconselhamos que na aplicação deste instrumento no âmbito da investigação, sejam utilizados os factores como índices independentes e não a nota global da escala.

No seu conjunto, a RSRI revelou boas características para ser utilizada em investigação e clínica com adolescentes. Contrariamente aos adultos, os jovens parecem distinguir-se nos valores médios obtidos na RSRI, revelando as raparigas valores mais elevados de inibição (total) e de Medos/doenças, em geral, bem como de medos relacionados com a escola e situações sociais. Este resultado é apoiado por outros estudos que mostraram a tendência do género feminino para revelar valores mais elevados de inibição (Reznick, 1989; Scarpa, Raine, Venables, \& Mednick, 1995). Provavelmente, estes resultados reflectem estereótipos ligados aos papéis sexuais dominantes, que esperam que as raparigas sejam mais inibidas, e que os rapazes 
manifestem um comportamento menos inibido. Dois estudos com crianças, contudo, não encontraram resultados relativos à diferença entre os géneros (Broberg et al., 1990; Kagan \& Snidman, 1991). Já em relação à idade, serão necessários mais estudos que clarifiquem a eventual incidência desta variável, uma vez que no nosso estudo o efeito é modesto.

Relativamente à comparação entre os três grupos, constatámos que os fóbicos sociais se distinguem dos normais na forma como avaliam a sua inibição comportamental durante a infância, evidenciando valores significativamente mais elevados na medida global de inibição e nos componentes de medos sociais/escola e de medos e doenças (medos não sociais). O mesmo é dizer que os adolescentes com fobia social, comparativamente com os normais, percepcionam-se como tendo sido, na sua infância, mais receosos, com mais experiências de desconforto e evitamento de situações novas, com mais medos relacionados com a escola e mais medos e doenças em geral.

Quando comparados com o grupo misto de perturbações ansiosas, os fóbicos sociais continuam a distinguir-se na medida global de inibição comportamental, e na dimensão de medos sociais, mas não na dimensão de medos não-sociais, o que aponta para um efeito específico destes índices. Já a dimensão de medos e doenças (medos não-sociais), ao não distinguir o grupo de fóbicos sociais do grupo misto de ansiosos, sugere ser um factor de vulnerabilidade comum aos dois grupos clínicos. Estes resultados estão, no seu conjunto, em consonância com os obtidos em outros estudos semelhantes, pesem embora pequenas diferenças relativas aos desenhos das investigações em causa (Hayward et al., 1998; Mick \& Telch, 1998; Van Ameringen et al., 1998). Estes autores procuraram evidenciar a utilidade bidimensional do constructo de inibição comportamental, tal como é avaliado pela RSRI, mostrando a dimensão de medos sociais maior poder discriminativo do que a dimensão de medos não-sociais. O componente da inibição comportamental relativo aos medos sociais parece ser especialmente preditor da ansiedade social, corroborando, assim, a ideia que este tipo de medos na infância pode exacerbar ou criar condições para aumentar os níveis de ansiedade social.

Mais investigações são necessárias para clarificar a relação entre a inibição comportamental e as perturbações ansiosas específicas, justificando-se o seu estudo em amostras maiores, seguidas longitudinalmente e com controlo das situações comórbidas, nomeadamente de ansiedade social, que possam estar a influenciar os resultados. Não obstante as boas características psicométricas encontradas na presente investigação, torna-se importante em futuros estudos confirmar os resultados obtidos, bem como avaliar a replicabilidade das memórias, isto é, a análise da validade posditiva. Neste sentido, um próximo estudo poderá envolver a avaliação dos pais quanto à inibição dos seus filhos e comparar o grau de concordância entre estes dois pontos de vista. 


\section{Referências bibliográficas}

Biederman, J., Hirshfeld-Becker, D. R., Rosenbaum, J. F., Herot, C., Friedman, D., Snidman, N., Kagan, J., \& Faraone, S. V. (2001). Further evidence of association between behavioral inhibition and social anxiety in children. American Journal of Psychiatry, 158(10), 1673-1679.

Broberg, A. (1993). Inhibition and children's experiences of out-of-home care. In K. H. Rubin \& J. Asendorpf (Eds.), Social withdrawl, inhibition, and shyness in childhood (pp. 151-176). Hillsdale, N.J.: L. Erlbaum Associates.

Broberg, A., Lamb, M. E., \& Hwang, P. (1990). Inhibition: Its stability and correlates in sixteen- to forty-month-old children. Child Development, 61(4), 1153-1163.

Cunha, M. (2005). Ansiedade social na adolescência: Avaliação e trajectórias de desenvolvimento (Social anxiety in adolescence: Assessment and developmental paths). Unpublished Doctoral thesis, Coimbra University, Coimbra.

Cunha, M., Soares, I., \& Pinto-Gouveia, J. (2008). The role of individual temperament, family and peers in social anxiety disorder - a controlled study. International Journal of Clinical and Health Psychology, 8(3), 631-655.

de Figueiredo, J. M. (1980). Some methodological remarks on transcultural interviewing on psychopathology. International Journal of Social Psychiatry, 26(4), 280-292.

DiLalla, L. F., Kagan, J., \& Reznick, J. S. (1994). Genetic etiology of behavioral ihnibition among two-year-old children. Infant Behavior and Development, 17, 401-408.

Floyd, F. J., \& Widaman, K. F. (1995). Factor analysis in the development and refinement of clinical assessment instruments. Psychological Assessment, 7, 286-299.

Garcia-Coll, C., Kagan, J., \& Reznick, J. S. (1984). Behavioral inhibition in young children. Child Development, 55, 1005-1019.

Hayward, C., Killen, J. D., Kraemer, H. C., \& Taylor, C. B. (1998). Linking self-reported childhood behavioral inhibition to adolescent social phobia. Journal of the American Academy of Child \& Adolescent Psychiatry, 37(12), 1308$-1316$.

Kagan, J. (2001). Temperamental contributions to affective and behavioral profiles in childhood. In S. G. Hofmann \& P. M. DiBartolo (Eds.), From social anxiety to social phobia: multiple perspectives (pp. 216-234). Boston, MA: Allyn and Bacon.

Kagan, J., Arcus, D., Snidman, N., Feng, W., Hendler, J., \& Greene, S. (1994). Reactivity in infants: A cross-national comparison. Developmental Psychology, 30(342-345).

Kagan, J., Reznick, J. S., \& Gibbons, J. (1989). Inhibited and uninhibited types of children. Child Development, 60(4), 838-845. 
Kagan, J., Reznick, J. S., \& Snidman, N. (1988). Biological bases of childhood shyness. Science, 240(4849), 167-171.

Kagan, J., \& Snidman, N. (1991). Temperamental factors in human development. American Psychologist, 46(8), 856-862.

Mick, M. A., \& Telch, M. J. (1998). Social anxiety and history of behavioral inhibition in young adults. Journal of Anxiety Disorders, 12(1), 1-20.

Mineka, S., \& Zinbarg, R. (1995). Conditioning and ethological models of social phobia. In R. Heimberg, M. Liebowitz, D. Hope \& F. R. Schneier (Eds.), Social Phobia: Diagnosis, assessment, and treatment (pp. 134-162). New York: Guilford Press.

Neal, J. A., Edelmann, R. J., \& Glachan, M. (2002). Behavioural inhibition and symptoms of anxiety and depression: Is there a specific relationship with social phobia?. British Journal of Clinical Psychology, 41(4), 361-374.

Pais-Ribeiro, J. (1999). Investigação e avaliação em Psicologia e Saúde. Lisboa: Climepsi.

Reznick, J. S. (1989). Perspectives on behavioral inhibition. Chicago: University of Chicago Press.

Reznick, J. S., Hegeman, I. M., Kaufman, E. R., Woods, S. W., \& Jacobs, M. (1992). Retrospecive and concurrent self-report of behavioral inhibition and their relation to adult mental health. Developmental Psychology, 4, 301-321.

Reznick, J. S., Kagan, J., Snidman, N., Gersten, M., Baak, R., \& Rosenberg, A. A. (1986). Inhibited and uninhibited children: A follow-up study. Child Development, 57, 660-680.

Scarpa, A., Raine, A., Venables, P. H., \& Mednick, S. A. (1995). The stability of inhibited/uninhibited temperament from ages 3 to 11 years in Mauritian children. Journal of Abnormal Child Psychology, 23(5), 607-618.

Schwartz, C. E., Snidman, N., \& Kagan, J. (1999). Adolescent social anxiety as an outcome of inhibited temperament in childhood. Journal of the American Academy of Child \& Adolescent Psychiatry, 38(8), 1008-1015.

Silverman, W. K., \& Albano, A. M. (1996). Anxiety Disorders Interview Schedule for DSM-IV: Child version. Child Interview Schedule. USA: Graywind Publications Incorporated.

Suomi, S. (1986). Anxiety-like disorders in young nonhuman primates. In R. Gittelman (Ed.), Anxiety disorders of childhood (pp. 1-23). New York: Guilford Press.

Van Ameringen, M., Mancini, C., \& Oakman, J. M. (1998). The relationship of behavioral inhibition and shyness to anxiety disorder. Journal of Nervous Mental Disease, 186(7), 425-431. 